were included in this study. Before enrolment into the study, the patients were explained about the study and informed consent was obtained. The patients with unidentified colitis were excluded. The data on demographics, disease characteristics, FI (Vaizey score), and quality of life (IBD-Q) were collected. Data were analyzed using SPSS version 21.

Results There were 184 patients (women $=101,54.9 \%$; UC $=153,83.2 \%$ ) with a female preponderance for UC (male/ female ratio $=1: 1.5$ ) and a male preponderance for CD (male/female $=2: 1$ ). Forty-eight $(26 \%)$ patients reported symptoms of FI. Among the patients with FI, 70.8\% were women $(\mathrm{n}=34)$ and $29.2 \%$ were men $(\mathrm{n}=14)$ with an average age of 52.7 years (range, 20-78 years). Average age of onset of FI was 48.6 (range, 22-74) years. Ten percent (n $=5$ ) reported regular FI. Incontinence to flatus was seen in $33.3 \%(\mathrm{n}=16)$, to liquid faeces in $56.2 \%(\mathrm{n}=27)$, to solid faeces in $6.2 \%(n=3)$ and to all three in $4.1 \%(n=2)$. Twenty-one percent $(\mathrm{n}=10)$ complained of disruption of their physical and social activity. There was no association between FI and type of IBD. Significant associations were found between FI and age $(\mathrm{P}=0.005)$ and gender $(\mathrm{P}<$ 0.001). QOL in our cohort of patients was significantly affected by FI.

Conclusions In our study, nearly a quarter of patients reported FI. There was a significant correlation between FI and QOL. Therefore, enquiring about FI in IBD patients can lead to identification of this debilitating condition. This will enable early referral for continence care in this group of patients.

\section{P160 PRE-BIOLOGIC SCREENING IN A HIGH RISK AREA: ARE WE ADHERING TO GUIDELINES?}

Eilis Kempley, Rajan Pooni, Cheh Kuan Tai*, Noor Jawad. Newham University Hospital, London, UK

\subsection{6/gutjnl-2020-bsgcampus.235}

Introduction Newham University Hospital, Bart's NHS Trust, serves the London Borough of Newham which had the highest incidence of tuberculosis in the UK at 78.0 per 100,000 in 2014. Newham also has the highest average annual rate of new reported acute hepatitis $B$ infection in the UK. There have been clear guidelines on pre-biologic screening for opportunistic infections since 2014. Our aim is to assess whether patients who are on biologic therapy have been appropriately screened prior to initiation of biologic therapy.

Methods A retrospective review of all IBD patients on the biologic database was performed in November 2019. Patients who were initiated on biologics prior to the publication of guidelines in June 2014 were excluded.

Results The total number of patients was 63. 31 patients (49.2\%) had latent tuberculosis testing with Interferon-gamma release assay (IGRA) testing and 2 were positive. Screening with Chest XR (CXR) was better with 58.9\% concordance. 36 patients had normal CXRs and 1 had an appearance of a granuloma.

In comparison, viral screening had higher completion rates. Hepatitis B Surface Antigen (HbsAg) was sent in 53 patients $(84 \%)$ and all were negative. Hepatitis B Core Antibody $(\mathrm{HbcAb})$ was sent in 23 patients $(36.5 \%)$ and 1 was positive.

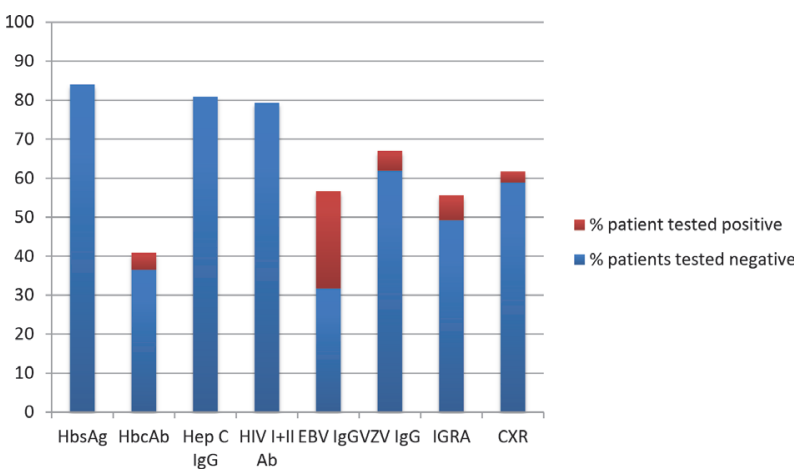

Abstract P160 Figure 1 Percentage of patients who received respective pre-biologic test

1 patient was $\mathrm{HbcAb}$ positive but $\mathrm{HbsAg}$ negative. In terms of Hepatitis C, 51 patients (80.9\%) had Hepatitis C IgG sent and all were negative. All 50 patients (79.3\%) who were tested for Human immunodeficiency virus (HIV I+II antibody) were negative. Ebstein Bar Virus IgG was sent in 20 patients (31.7\%), out of which, 15 were negative. Varicella-Zoster Virus IgG was sent in 39 patients $(61.9 \%)$ and 2 were positive.

An infection history was not taken, for either bacterial, fungal or viral infections and Bacille Calmette-Guerin vaccination status was not documented. No documentation was present regarding measles status. Routine vaccination status was not confirmed for diphtheria, poliomyelitis, pertussis, tetanus or Human Papilloma Virus. Prior to initiation of immunomodulation, vaccination was not considered for pneumococcal or influenza infections.

Conclusions Despite suboptimal pre biologics screening in this high risk region of East London for Tuberculosis and Hepatitis $\mathrm{B}$, no cases of reactivation of either Tuberculosis or Hepatitis $\mathrm{B}$ have been identified to date. The results suggest that clinicans are requesting some tests but not all. Following on from these results, we will be streamlining the process for ensuring all tests are performed prior to biologic initiation with a checklist proforma for the patients notes and on our biologics database for all prescribing gastroenterologists, as per ECCO guidelines.

\section{P161 IKK $\alpha$ AS A POTENTIAL THERAPEUTIC TARGET FOR THE PREVENTION OF INFLAMMATORY BOWEL DISEASE}

${ }^{1}$ Joseph Tang*, ${ }^{1}$ Stamatia Papoutsopoulou, ${ }^{2}$ Andrew Paul, ${ }^{2}$ Professor Simon Mackay, ${ }^{1}$ Carrie Duckworth, ${ }^{1}$ Mark Pritchard. ${ }^{1}$ University Of Liverpool, Liverpool, UK; ${ }^{2}$ University fo Strathclyde, Glasgow, UK

\subsection{6/gutjnl-2020-bsgcampus.236}

Introduction Current treatment options for inflammatory bowel disease (IBD) are primarily designed to suppress an established inflammatory response, but they do not prevent the initiation of the inflammatory cascade. We have previously demonstrated that the NF- $\mathrm{KB}$ signalling pathways play a pivotal role in murine experimental models of IBD. In particular, we showed that $n f \kappa b 2-/-$ mice were protected against LPSinduced cell shedding and DSS-induced colitis compared to wild-type mice. Specifically, our data suggested that NF-кB2 signalling in intestinal epithelial cells played a more important 